\title{
A Data Driven Model for the Detection of Random Waypoint
}

\author{
Ting Wang, Senior Member, IACSIT and Chor Ping Low
}

\begin{abstract}
Locational data are extremely useful resource to study customer behavior and mobility patterns. In this paper, beyond directly measuring how their location, velocity and acceleration change over time, we extend our discussion to construct a data driven model to quantitatively evaluate the moving objects' interests and intentions, which are represented by their waypoints distributions. Waypoints are defined with the Random Waypoint (RWP) mobility model, which is one of the most commonly used models in mobility management. To effectively deploy RWP model, the detection of accurate waypoint distribution is crucial and, however, challenging in most practical situations. Moreover, to understand the how and why an object moves in a its specific pattern, the knowledge of waypoint distribution could be valuable in many use cases. In this work, we analytically derive the relationship between waypoint distribution and the locational data that could be obtained directly from sensors, such as the number of objects' arrivals to a particular area. An estimation scheme using supervised learning algorithm is proposed to simplify the evaluation of our model. Simulations are carried out to verify the correctness and accuracy of our proposed scheme.
\end{abstract}

Index Terms-Locational data, mobility management, waypoint distribution, supervised learning.

\section{INTRODUCTION}

With the advances in sensor technologies and positioning systems (such as GPS and aGPS etc.), locational data are becoming pervasive in our daily lives and scientific researches. Either indoor or outdoor, it is not difficult to obtain the trace, the velocity, and even the acceleration of any moving entity (referred to as an object in this paper) of our interest, providing proper equipment and infrastructure. Massive data have been collected in various research projects since early 90's [1]. As part of the "big data regime", interests in locational data have recently grown even more rapidly thanks to the new database technology and data mining techniques. With the ability to store and analyze data with tremendous size, researchers and stake holders are no longer satisfied with obtaining and retaining the data that are directly measurable from movement of the object itself (e.g. position, velocity, direction etc.), and the focus has been shifted to finding out the information hidden underneath the numbers (a.k.a. locational data mining [2] or trajectory

Manuscript received February 20, 2013; revised June 21, 2013. This work was supported in part by the Singapore Economic Development Board (EDB).

Wang Ting is now with SAP Asia Ptd Ltd, Singapore (e-mail: dean.wang@sap.com).

Chor Ping Low is with School of Electrical and Electronic Engineering, Nanyang Technological University, Singapore (e-mail: icplow@ntu.edu.sg). pattern mining [3]), such as distribution and spatial-temporal trending of the moving entities so that proper services could be offered, and more effective strategies could be executed accordingly [4]. In this paper, we study the locational data and derive a quantitative representation of the objects' interests and intentions - namely the waypoint distribution — with a supervised learning model [5].

Waypoint is defined in the Random Waypoint (RWP) mobility model, which is first used by Johnson and Maltz in their study of Dynamic Source Routing (DSR) [6] and then becomes a benchmark model to evaluate the routing protocols and other wireless mobile communication applications. Briefly, an object with the RWP mobility moves along a zigzag line from one point to another inside a given area. These points are referred to as waypoints and the path between two consecutive waypoints is defined as a leg. For passive moving entities, waypoints can be used to control their movement [7]; for pro-active moving entities, their decisions of moving around would be reflected by how the waypoints are distributed in the area.

Obviously, many real life scenarios other than wireless communications can be modeled by RWP. For example, in a conference, people do not walk randomly - they move from one discussion group to another, or move to the buffet counter to get some food. The locations of discussion groups and buffet counters can be considered as waypoints. Military vehicles on an open battle field usually move on certain direction for a period of time before changing direction again. Similarly, in a soccer match, players either chase the ball or move to flanks to explore opportunities. The movement of the player could be broken into legs that reflect his/her decision of moving from one place to another. Because of its simplicity, wide adaptability and good flexibility, RWP has soon been extended to other fields of research, such as robotics [8] and customer behavior studies [9].

In the conventional RWP, the waypoints are uniformly distributed over the entire area. However, the "uniform" assumption can hardly be satisfied in most real life cases. Certain regions could be more attractive and worth moving to comparing to others. Such regions are referred to as the hot-spots. For example, in a conference, participants maybe attracted by different discussion groups, buffet tables, or poster areas, instead of other random locations. The waypoint distributions are therefore not uniformly distributed over the entire meeting venue. Or consider the battlefield example, the headquarters and warehouses attract more vehicles to move to. In the soccer match, the locations of "hot-spots" are related to the strategy of the team and the role of the player. Different players will have different hot-spots to move towards to and thus different waypoint distributions. To 
address these phenomena, the Non-Uniform Random Waypoint (NU-RWP) model has been proposed by Hyytia et. al. in [10]. Distributions other than the uniform distribution can be used to generate the waypoints.

Given the waypoint distribution as known, useful insights can be revealed by many existing analytical models, as discussed in [11]-[13]. However, waypoint distribution may not be directly measured or told. Using sensors or detectors, we could measure the frequency of hosts arriving/leaving certain area, the time the hosts spent in certain area, but these are not equivalent to the distribution of waypoints. Waypoints distribution is abstract and difficult to obtain, because even the objects themselves (e.g. people in the conference and soccer players etc.) may not be aware of how exactly their waypoints are distributed. They — on their own - may not be able to define their interests and intentions with some values. But they can be examined and evaluated with the waypoint distribution. In some other cases, such as the battle field example, waypoint distribution may be deliberately hidden from others so that important military information would not be leaked. In order to model and study the behavior of the mobile objects, waypoint distributions need to be studied. In addition, if we are able to detect waypoint distribution, we will be able to find out which discussion groups (or buffet tables) are more attractive in the conference; where the warehouses or headquarters locate in the battle field; and whether a certain soccer player is being more attacking or defensive in his/her mind, in the previous examples. In other words, it becomes possible that we represent people's minds (interest, intentions, or even sub-conscious) using a distribution function that could be quantitatively evaluated. This information could be of great strategic value, and thus a model to derive waypoint distribution would be needed.

In this paper, we take the challenge to find out the waypoint distribution from other properties that are directly detectable by sensors. In particular, we use a variable namely arrival rate $\gamma$, which can be easily derived from locational data, to train a supervised learning system to obtain an estimation of the waypoint distribution. We introduce the background of our discussion in Section II. The theoretical analysis of the relationship between waypoints distribution and arrival rate $\gamma$ is discussed in Section III, together with an estimation scheme. The correctness of this scheme is verified through simulations in Section IV. Section V demonstrates how our model can be deployed with some examples. Finally, Section VI concludes this paper.

\section{PRELIMINARIES}

In this section, we will introduce the preliminary definitions and assumptions before we present our waypoints distribution detection scheme.

\section{A. Non-Uniform Random Waypoint Mobility Model}

In NU-RWP, the waypoints are generated according to a certain probability density function (pdf) $f(x, y)$, which may not be uniform. We note that $f(x, y)$ can be either continuous or discrete, as shown in Fig. 1. Moreover, it can also be in a polar coordinate system, $f(\theta, r)$, e.g. in Fig. 1 (b), $f(\theta, r)=\frac{3 r^{2}}{2 \pi a^{3}}$. We use $f(x, y)$ to denote the pdf of the distribution of a host by default in this work. In fact all the analysis applies to polar coordinates as well.

The probability of a waypoint being generated in a given area $A$ is

$$
F(A)=\iint_{\mathrm{A}} f(x, y) d x d y
$$

Those areas with higher values of $F(A)$ are the hotspots, shown by the darker shaded areas in Fig. 1, meaning such places are more favorable than the others. In contrast, those with extreme low $F(A)$ values could be referred to as cold-spots, which are the regions with pale color in Fig. 1. The "hotness" and "coldness" reflect the objects' willingness of moving to such places. Moreover, the value of $F(A)$ would be a quantitative definition of the objects' interests and intentions for area $A$.

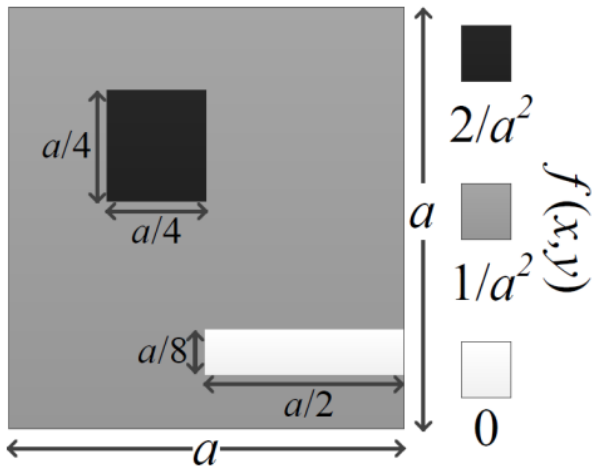

(a) Discrete Distribution

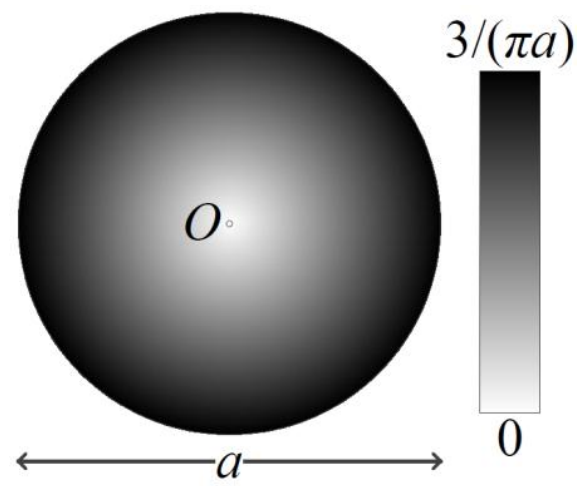

(b) Continuous Distribution

Fig. 1. Different types of waypoints distributions.

We assume that the movement of all the objects is restricted inside a convex area denoted as $A^{0}$, thus we have $\int_{A^{0}} f(x, y) d x d y=1$. To estimate $f(x, y)$, we can evaluate the probabilities of the waypoints being generated in different parts of $A^{0}$.

It is well known with RWP model that the arrivals of multiple independently moving objects form a Poisson process [14], and it can thus be decomposed to arrivals of each single host according to the superposition property and vice versa. For the convenience of discussion, we consider a 
single object first. The discussion could easily be extended to a scenario with multiple objects.

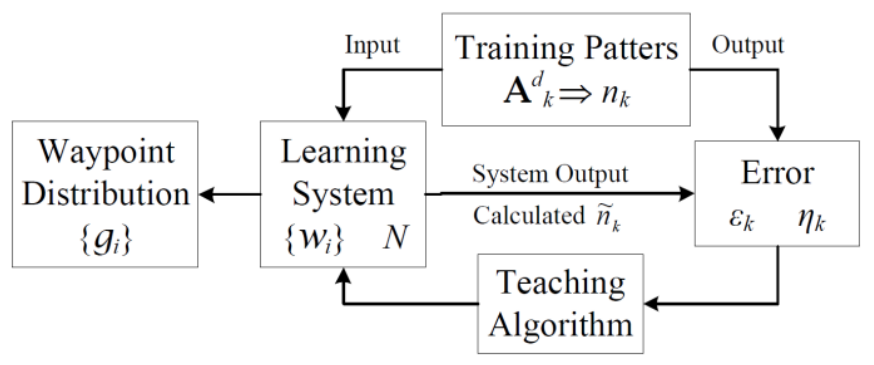

Fig. 2. Overview of supervised learning system.

\section{B. Supervised Learning System}

The block diagram of supervised learning system [5] is described through Fig. 2. Training patterns consist a number of input-output pairs are used to teach the learning system, which takes the training input and produces system output. The teaching algorithm processes the errors constructed from the difference between the desired output (from training patterns) and system output to update the learning system. Through repeated interactive training procedures, the parameters in the learning system are adjusted by the error in a way that the system output tries to follow the desired output as close as possible.

In our work, during a certain period of time, we measure the number of times that the object enters (arrives) a given concave area (referred to as destination area $A^{d}$ ) inside $A^{0}$. We use $n$ to denote the number of arrivals of the object to a particular $A^{d}$.

To find the waypoint distribution of the object, we need to consider multiple destination areas $\left\{A_{k}^{d}\right\}$ and corresponding number of arrivals $\left\{n_{k}\right\}$. The data pairs $\left\{\left(A_{k}^{d}, n_{k}\right)\right\}$ will be our training patterns.

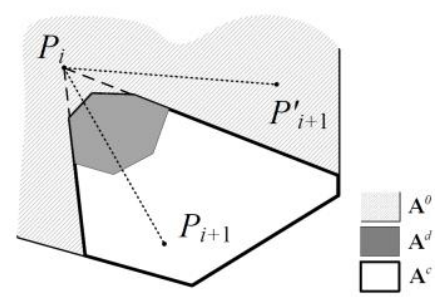

Fig. 3. Corresponding area definition.

From input, the vertex coordinates of $\left\{A_{k}^{d}\right\}$ and the total number of legs $N$, the learning system will use the model we derive in Section III to calculate the estimated number of arrivals, denoted as $\tilde{n}_{k}$. The error between $\tilde{n}_{k}$ and $n_{k}$ (consists of the absolute error $\varepsilon_{k}$ and the relative error $\eta_{k}$ ) will then be processed by our teaching algorithm discussed in Section III-D in order to update the parameters in the learning system $\left\{w_{i j}\right\}$. The values of $\left\{w_{i j}\right\}$ will be used to determines the distribution of waypoints represented by $\left\{g_{j}\right\}$. The meaning and usage of these variables will be introduced in the following sections.

\section{Distribution DeteCtion SCHEME}

To derive the waypoints distribution, we define and evaluate the following attributes:

1) Probability of Arrival: the probability of the object arriving in $A^{d}$ via a particular leg $i$, denoted as $\operatorname{Pr}_{i}$;

2) Arrival Rate: the expected probability that the object arriving in $A^{d}$ in a randomly given leg, denoted as $\gamma$. Using the mathematical property of the geometric model, we design the following algorithms to evaluate

We denote the $i^{\text {th }}$ waypoint of the object as $P_{i}$.

\section{A. Evaluation of $\operatorname{Pr}_{i}$}

When the object moves on the $i^{\text {th }}$ leg (denoted as $\ell_{i}$ ), it arrives in $A^{d}$ only if the directional line segment $P_{i} P_{i+1}$ intersects with $A^{d}$. This is a necessary but not sufficient condition, because when $P_{i}$ is inside $A^{d}$, the object will either stay inside the destination area $A^{d}$ or move out of $A^{d}$. We define another new term, namely the corresponding area of a given point $P$ and a destination area $A^{d}$, denoted by $A^{c}\left(P, A^{d}\right)$ as follows:

- if $P_{i}$ is at $P$ and $P_{i+1}$ is located in the corresponding area of $P$, the object will arrive in $A^{d}$ in $\operatorname{leg} \ell_{i}$ (i.e. $P_{i} P_{i+1}$ intersects with $A^{d}$ and $P$ is not inside $A^{d}$ );

- Otherwise, if $P_{i+1}$ is located outside the corresponding area, the object will not arrive in $A^{d}$ in $\ell_{i}$.

Understanding that $A^{c}\left(P, A^{d}\right)$ can be calculated from the coordinates of $P$ and $A^{d}$ vertices, we abbreviate the notation to $A^{c}$.

In Fig. 3 we show that if $P_{i+1}$ is within the corresponding area $A^{c}, \operatorname{leg} \ell_{i}$ would pass through the destination area; while as $P_{i+1}^{\prime}$ is located outside the corresponding area, $\ell_{i}$ would not pass through $A_{d}$, meaning that object $\mathrm{k}$ will not arrive in the destination area in leg $\ell_{i}$. We can see that the corresponding area $A^{c}$ is bounded by the two tangent lines of $A^{d}$ from $P$ and the edges of $A^{d}$ and $A^{0}$, as shown in Fig. 3.

Therefore, the probability of the object arriving in $A^{d}$ via leg $\ell_{i}$ is equivalent to the probability that $P_{i+1}$ is located within $A^{c}$ which - according to Eqn. 1 - can be evaluated as:

$$
\operatorname{Pr}_{i}=F\left(A^{c}\right)=\iint_{\mathrm{A}^{c}} f(x, y) d x d y
$$

\section{B. Evaluation of $\gamma$}

The value of $\gamma$ can be estimated empirically as $\gamma=\frac{n}{N}$, where $N$ is the total number of legs that the object has traveled in an adequately long period of time, and $n$ is the number of the object's arrivals to the area $A^{d}$ during this period. Since with RWP, each waypoint is independently 
generated, $\operatorname{Pr}_{i}$ is independent from $\operatorname{Pr}_{j}$, if $i \neq j$. Considering that there are $N$ legs, we can estimate the number of arrivals $n$ by

$$
n \approx \operatorname{Pr}_{1}+\operatorname{Pr}_{2}+\ldots+\operatorname{Pr}_{N}=\sum_{i=1}^{N} \operatorname{Pr}_{i}
$$

Under the assumption that the object has traveled in an adequately long period of time, $N$ should be adequately large. The arrival rate can be represented as

$$
\gamma=\frac{n}{N}=\frac{1}{N}\left(\sum_{i=1}^{N} \operatorname{Pr}_{i}\right)
$$

It indicates that $\gamma$ is in fact the expected value of $\operatorname{Pr}_{i}$, denoted as $\overline{\operatorname{Pr}_{i}}$. Therefore,

$$
\begin{aligned}
\gamma & =\overline{\operatorname{Pr}_{i}}=\iint_{P_{i} \in \mathrm{A}^{c}} f\left(x_{P_{i}}, y_{P_{i}}\right) \operatorname{Pr}_{i} d x_{P_{i}} d y_{P_{i}}=\frac{n}{N} \\
& =\iint_{P \in \mathrm{A}^{n}} f\left(x_{P}, y_{P}\right)\left(\iint_{\mathrm{A}^{c}} f(x, y) d x d y\right) d x_{P} d y_{P}
\end{aligned}
$$

Thus we have

$$
n=N \iint_{P \in \mathrm{A}^{n}} f\left(x_{P}, y_{P}\right)\left(\iint_{\mathrm{A}^{c}} f(x, y) d x d y\right) d x_{P} d y_{P}
$$

Theoretically, the above equation could be used as the supervised learning system, because from the vertices coordinates of $A^{d}$, we are able to locate $A^{c}\left(P, A^{d}\right)$ and thus evaluate $n$. However, solving these integrations could be tedious; we propose the following estimation scheme to simplify the process.

\section{Grid-Based Distribution Estimation}

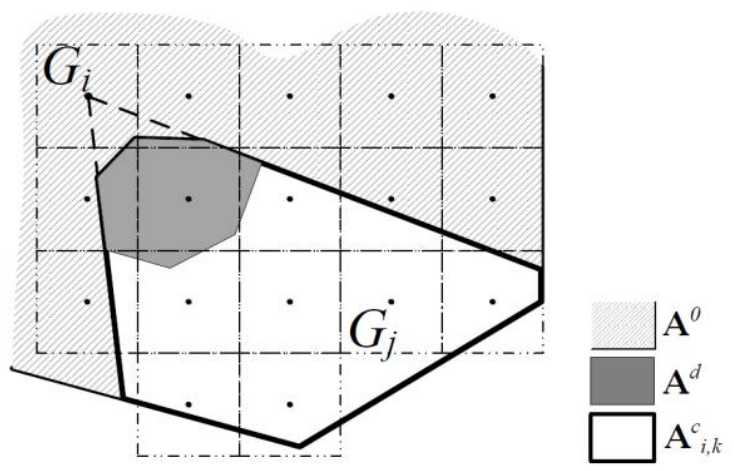

Fig. 4. Corresponding area with grids.

We put the network area into a grid graph. $G_{i}$ denotes the center of grid $i$ as shown in Fig. 4. We assume that there are $N_{g}$ grids of which the centers are inside $A^{d}$. We assign a real positive number $g_{i}$ to each $G_{i}$ so that

$$
\begin{aligned}
g_{i} & =\operatorname{Pr}[\text { a waypoint is generated inside grid } i] \\
& =\int_{G_{i}} f(x, y) d x d y
\end{aligned}
$$

and

$$
\sum_{G_{i} \in \mathrm{A}^{n}} g_{i}=\sum_{i=1}^{N_{g}} g_{i}=1
$$

We can thus estimate $f(x, y)$ by evaluating $\left\{g_{i}\right\}$.

Consider a number of $N_{d}$ destination areas of our interest, they can be $N_{d}$ sensors deployed inside $A^{0}$ with their sensing range as $A^{d}$ 's; or simply areas we select to study the trace of the objects. A corresponding area $A^{c}\left(G_{i}, A_{k}^{d}\right)$ (denoted as $\mathrm{A}_{i k}^{c}$ for short in the next equation) can be defined with respect to $G_{i}$ and $A_{k}^{d}$. We use $n_{k}$ to denote the number of times the object arrives at $A_{k}^{d}$. Based on Eqn. 4:

$$
n_{k}=N \sum_{i=1}^{N_{g}}\left(g_{i} \sum_{G_{j} \in \mathrm{A}_{i k}^{c}} g_{j}\right)=N \sum_{i=1}^{N_{g}} \sum_{G_{j} \in \mathrm{A}_{i k}^{c}} g_{i} g_{j} .
$$

We note that since a point cannot be in its own corresponding area no matter where is the destination area, in the above equation we have $i \neq j$. We define a variable

$$
w_{i j}=\left\{\begin{array}{cc}
g_{i} g_{j} & \text { if } i \neq j \\
0 & \text { if } i=j
\end{array},\right.
$$

and a binary relationship $\mathbb{R}_{k}$ :

$$
(i, j) \in \mathbb{R}_{k} \Leftrightarrow G_{j} \in \mathrm{A}^{c}\left(G_{i}, \mathrm{~A}_{k}^{d}\right) .
$$

The expression of $n_{k}$ can be simplified to

$$
n_{k}=N \sum_{(i, j) \in \mathbb{R}_{k}} w_{i j}
$$

We have $w_{i j}=w_{j i}$ and $g_{i^{\prime}}=\frac{w_{i^{\prime} j}}{w_{i j}} g_{i}$ for arbitrary non-equal $i, j$ and $i^{\prime}$. Thus

$$
\sum_{i=1}^{N_{g}} g_{i}=\sum_{i^{\prime} \neq i} g_{i^{\prime}}+g_{i}=\left(\sum_{i^{\prime} \neq i, i^{\prime} \neq j^{*}} \frac{w_{i^{\prime} j^{*}}}{w_{i j^{*}}}+1\right) g_{i}=1,
$$

where the values of $j^{*}$ can be different in different terms. For example, if $i=1$, we can use different value of $j *$ to evaluate $\sum_{i^{\prime} \neq i} \frac{w_{i^{\prime} j^{*}}}{w_{i j^{*}}}=\frac{w_{2,5}}{w_{1,5}}+\frac{w_{3,2}}{w_{1,2}}+\frac{w_{4,7}}{w_{1,7}}+\cdots+\frac{w_{N_{g} j^{*}}}{w_{1 j^{*}}}$, where $j^{*}=5,2,7, \ldots$.

This equation shows that the values of $\left\{g_{i}\right\}$ and the 
$\left\{w_{i j}\right\}$ waypoints distribution can be derived if we can evaluate $\left\{w_{i j}\right\}$. Therefore, in the learning system, if the values of $\left\{w_{i j}\right\}$ and $N$ are estimated, $\left\{g_{i}\right\}$ can be easily derived from them, and thus the waypoint distribution.

\section{Teaching Algorithm}

The learning system is initialized as if the waypoints are uniformly distributed, where $g_{i}=\frac{1}{N_{g}}$ and thus $w_{i j}=\frac{1}{N_{g}^{2}}$. $N$ can be determined empirically. In our case it is initialized as $\tilde{N}=2 \max \left\{n_{a k}\right\}$.

In each iteration of the supervised training, $\tilde{n}_{a k}$ is computed from $\mathrm{A}_{k}^{d},\left\{w_{i j}\right\}$ and $\tilde{N}$ using Eqn. 5. As the values of $\left\{w_{i j}\right\}$ and $\tilde{N}$ we are using may not be accurate, the following errors could be produced:

- The Absolute Error: $\varepsilon_{k}=n_{k}-\tilde{n}_{k}$,

- The Relative Error: $\eta_{k}=\frac{n_{k}}{\tilde{n}_{k}}$.

They are used to update the values of $\left\{w_{i j}\right\}$ and $\tilde{N}$.

We first note that the learning system can be scaled by a factor of

$$
\mathrm{H}=\left(\prod_{k=1}^{N_{g}} \eta_{k}\right)^{\frac{1}{N_{g}}}
$$

Therefore, the value of $\tilde{N}$ can be updated as

$$
\tilde{N}^{*}=\tilde{N} \mathrm{H} \text {. }
$$

This step makes the value of $\tilde{N}$ converges quickly, as we will show in Section IV. It also proves the initial value of $\tilde{N}$ is not crucial in our algorithm, and thus can be determined empirically.

It will affect the value of absolute error $\varepsilon_{k}$, too:

$$
\varepsilon_{k}=n_{k} \mathrm{H}-\tilde{n}_{k}
$$

Therefore when we use $\left\{\epsilon_{k}\right\}$ and $H$ to update the values of $\left\{w_{i j}\right\}$, we have

$$
w_{i j}^{\prime}=w_{i j}+\sum_{k=1}^{N_{g}} \frac{\varepsilon_{k}}{\left|\mathbb{R}_{k}\right|},
$$

where $\left|\mathbb{R}_{k}\right|$ denotes the number of $(i, j)$ pairs in $\mathbb{R}_{k}$.

The values of $\left\{g_{i}\right\}$ can be obtained by Eqn. 6 as

$$
g_{i}=\left(\sum_{i^{\prime}=1, i^{\prime} \neq i, j^{*} \neq i^{\prime}}^{N_{g}} \frac{w_{i^{\prime} j^{*}}}{w_{i j^{*}}}+1\right)^{-1},
$$

Since we know that $\sum_{i=1}^{N_{g}} g_{i}=1$, we can normalize $\left\{g_{i}\right\}$ so that

$$
g_{i}^{*}=\frac{g_{i}^{*}}{\sum_{i=1}^{N_{g}} g_{i}^{*}}
$$

Then, $\left\{w_{i j}\right\}$ can be updated again as

$$
w_{i j}^{*}=g_{i}^{*} g_{j}^{*}
$$

The new values $\left(\left\{w_{i j}^{*}\right\}\right.$ and $\left.\tilde{N}^{*}\right)$ are used in the next iteration to compute the system output $\tilde{n}_{a k}$. This learning procedure repeats until the errors are close to zero. The values of $\left\{g_{i}\right\}$ are thus obtained, as we demonstrate in thenext section.

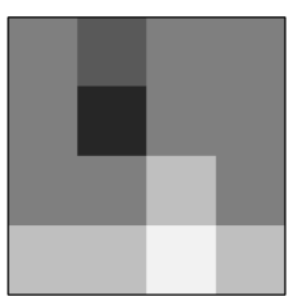

(a) Scenario 1: $N_{g}=16$

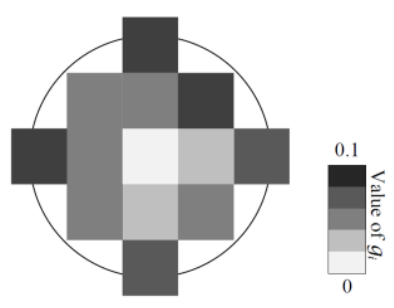

(b) Scenario 2: $N_{g}=13$ Fig. 5. Results with fewer sensors

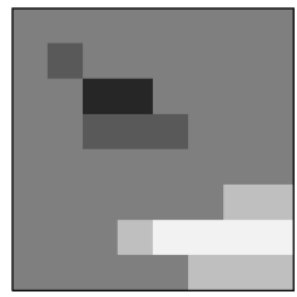

(a) Scenario 1: $N_{g}=64$

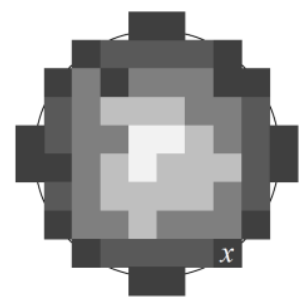

(b) Scenario 2: $N_{g}=68$

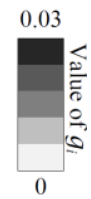

0
Fig. 6. Results with more sensors

\section{EXPERIMENT RESULTS}

We use the two examples in Fig. 1 to demonstrate the correctness of our scheme. The two scenarios are referred to as scenario 1 (Fig. 1 (a)) and 2 (Fig. 1(b) ) with $a=1000 \mathrm{~m}$. $\mathrm{C}++$ programs are used to simulate the object movement and measure the number of arrivals of different randomly selected destination areas. The average size of the destination areas is approximately $750 \mathrm{~m}^{2}$. The simulation duration length is $T=10000$ s.

Fig. 5 and Fig. 6 show our estimation of the waypoints distribution. The gray scaled color in each grid corresponds to its value of $\left\{g_{i}\right\}$. Comparing them with Fig. 1 where the exact distributions are depicted, we can see that the hot and cold-spots can be accurately identified in our results.

Since our estimation is grid-based, by having more grids, higher resolution of the waypoints distribution can be obtained. This is demonstrated by comparing Fig. 5 with Fig. 6 , where different numbers of grids are used. 


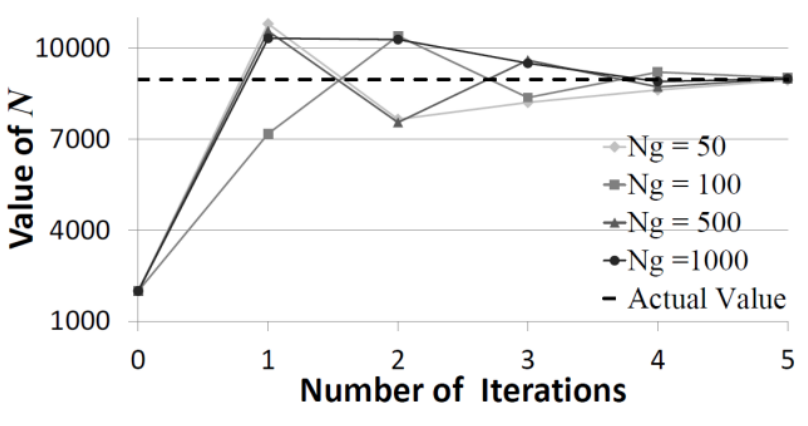

(a) $N$

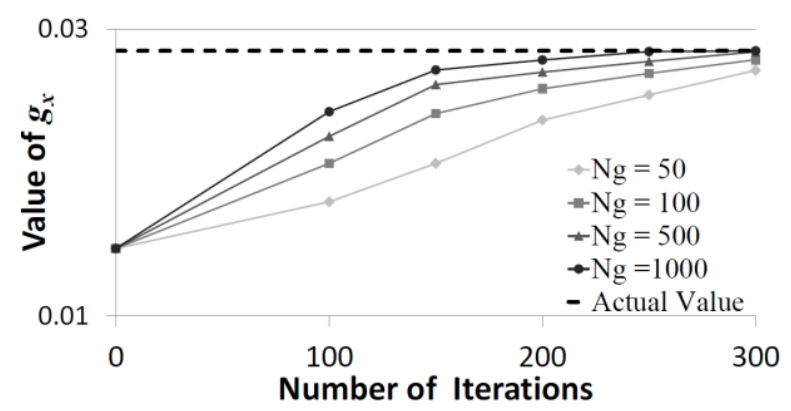

(b) $g_{x}$

Fig. 7. Progress of training.

The precision of estimation can be further improved if we train the system with more destination areas or go through more training iterations. This is demonstrated by Fig. 7. We consider a particular grid, namely grid $x$ in scenario 2 (at the lower right corner of Fig. 6 (b) ). We can see that $N$ and $g_{x}$ both eventually converge to the actual value (obtained from the actual distribution) with adequate number of iterations as depicted in Fig. 7 (a) and Fig. 7 (b), respectively. It can be observed that $N$ converges quickly towards the actual value, while value of $g_{x}$ changes much slower. This is because $N$ is scaled with the system in each iteration, and thus increases much faster than $g_{x}$, which is incrementally updated. We can see from Fig. 7(a) that when more sensors are deployed ( $N_{s}$ increases), there will be more training patterns available to teach the learning system, making each iteration more effective than the case with fewer sensors. The value of $g_{x}$ thus evolves much faster towards the actual values.

\section{USE CASE EXAMPLES}

To demonstrate how our model could be deployed for waypoint distribution detection, we propose three different examples on how data could be collected and destination areas could be selected:

1) Mobile sensors with detection capability are deployed. As the sensor move (not necessarily in RWP), they detect the number of arrivals of nearby objects. Thus the destination areas will be the regions within a sensor's sensing range. Even only one single sensor is used; it can collect data for multiple destination areas, as shown in Fig. 8 (a). Stationary sensors with directional antenna are deployed. It can change its sensing direction so that different destination area could be covered. Alternatively, an Omni-sensor with power controlling mechanism could also be deployed. Destination areas will be the concentric circles within the sensor's varying range, as depicted in Fig. 8 (b).

2) Sensors can be attached to the mobile objects of our interest to record their traces. In Fig. 8 (c), we show that it is possible to obtain their traces of the soccer players from the match first. Then destination areas could be defined manually and the waypoint distribution could be studied. We note in this scenario, since the complete movement trace is available, we may omit $N$ (total number of legs) in the learning system and simplify the training process.

Due to the constraint of page limits, we could not include any detailed case study in this paper. More use cases and examples will be demonstrated in an extended version.

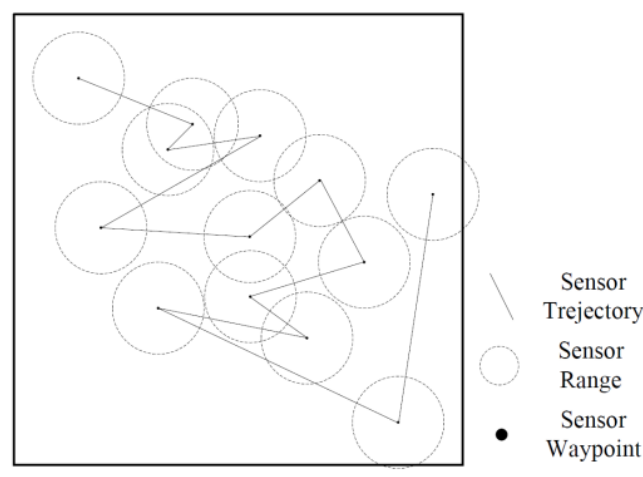

(a) Mobile Sensor

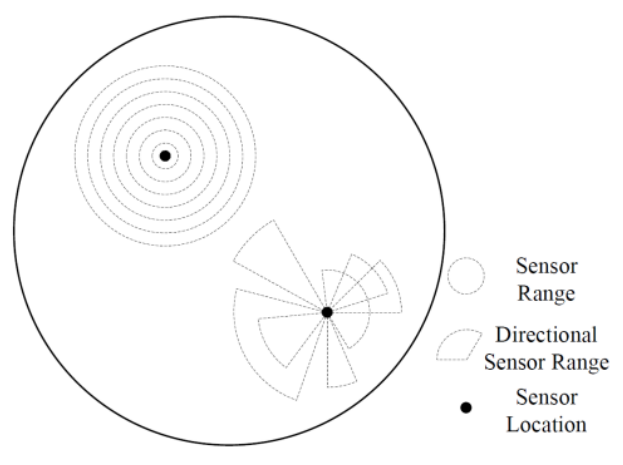

(b) Stationary Sensor

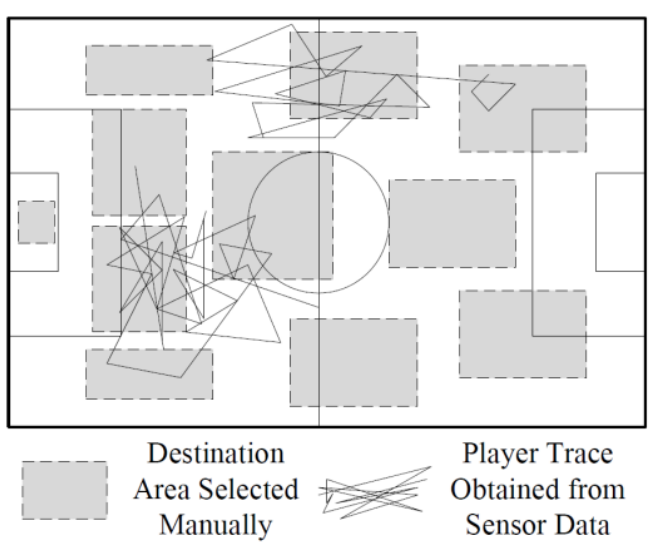

(c) Attached Sensor

Fig. 8. Case studies. 


\section{CONCLUSION}

In this paper, we construct a data driven, supervised learning system to detect waypoints distribution in mobility management problems. When the objects move in the Non-Uniform Random Waypoint mobility model, our model estimate how the waypoints are distributed from the numbers of arrivals of the objects to certain area. This information is of great practical value and can be used in various real life applications, such as customer behavior study, military surveillance, and sports data analysis. Moreover, waypoint detection would be a simple and intuitive way to evaluate the mobile objects' interest and preference when moving around the area. With the distribution function, people's intention and willingness could be quantitatively defined. Further studies could also be carried out based on this to address the reason that affects people's choice.

\section{REFERENCES}

[1] T. L. Nyerges, "Locational Referencing and Highway Segmentation in a Geographic Information System," ITE Journal, vol. 60, no. 3, pp. 27-31, 1990

[2] S. Shekhar, P. Zhang, and Y. Huang, "Spatial Data Mining," Data Mining and Knowledge Discovery Handbook, pp. 837-854, 2010.

[3] A. Monreale, F. Pinelli, R. Trasarti, and F. Giannotti, "WhereNext: a Location Predictor on Trajectory Pattern Mining," in Proc. the 15th ACM SIGKDD International conference on Knowledge Discovery and Data Mining (KDD'09), New York, NY, USA: ACM, 2009, pp. 637-646.

[4] D. Quercia, N. Lathia, F. Calabrese, G. D. Lorenzo, and J. Crowcroft, "Recommending Social Events from Mobile Phone Location Data," in Proc. IEEE 10th International Conference on Data Mining (ICDM 10'), pp. 971-976, Dec. 2010.

[5] B.-Y. Yaneer, Dynamics of Complex Systems, Westview Press, 2003.

[6] D. B. Johnson and D. A. Maltz, "Dynamic Source Routing in Ad Hoc Wireless Networks," Mobile Comput., vol. 353, pp. 153-181, 1996.

[7] T. Wang and C. P. Low, "A Fully Distributed Node Allocation Scheme with Partition Protection for Mobile Ad Hoc Networks," Computer Communications, vol. 33, no. 16, pp. 1949-1960, 2010.

[8] J. Bruce, M. Bowling, B. Browning, and M. Veloso, "Multi-Robot Team Sesponse to a Multi-Robot Opponent team," in Proc. the IEEE International Conference on Robotics and Automation, vol. 2, pp. 2281-2286, Sept. 2003.

[9] J. Krï ockel and F. Bodendorf, "Visual Customer Behavior Analysis at the Point of Sale," International Journal on Advances in Systems and Measurements, vol. 5, no. 3\&4, pp. 178-187, 2012.

[10] E. Hyyti"a, P. Lassila, and J. Virtamo, "Spatial Node Distribution of the Random Waypoint Mobility Model With Applications," IEEE Trans. Mobile Comput., vol. 5, no. 6, pp. 680-694, Jun. 2006.
[11] J.-Y. L. Boudec and M. Vojnovi'c, "Perfect Simulation and Stationarity of a Class of Mobility Models," in Proc. IEEE $24^{\text {th }}$ Annual Joint Conference of the IEEE Computer and Communications Societies (INFOCOM '05), vol. 4, pp. 2743-2754, 2005.

[12] P. Lassila, "Spatial Node Distribution of the Random Waypoint Mobility Model with Applications," IEEE Transactions on Mobile Computing, vol. 5, no. 6, pp. 680-694, member-Hyytia, Esa and Member-Virtamo, Jorma, 2006.

[13] T. Wang and C. P. Low, "Evaluating Inter-Arrival Time in General Random Waypoint Mobility Model," Ad Hoc Networks, vol. 11, no. 1, pp. 124-137, 2013.

[14] G. Sharma, R. Mazumdar, and N. B. Shroff, "Delay and Capacity Trade-offs in Mobile Ad Hoc Networks: A Global Perspective," IEEE/ACM Trans. Netw., vol. 15, no. 5, pp. 981-992, Oct. 2007.

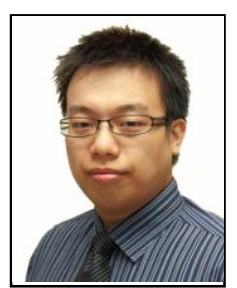

Wang Ting was born in 1983 in Chengdu, Sichuan, China. He came to Singapore for his undergraduate studies under Singapore Government-Linked Company SM2 scholarship in 2001. He obtained his Bachelor's degree with honors in 2005 and subsequently $\mathrm{Ph} . \mathrm{D}$ in 2011 both at Nanyang Technological University (NTU), Singapore.

He worked as a demand planner at Apple South Asia and joined SAP as a Data Scientist in 2012. His research interests include data mining, mathematical modeling and algorithmic optimization.

Dr. Wang loves soccer. He considers family as his greatest award. He has a son, and he is a good cook - said his wife.

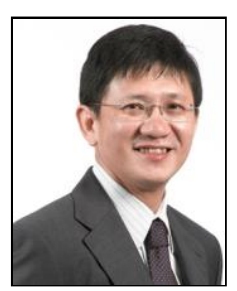

Low Chor Ping received his Ph.D. in computer science from the National University of Singapore (NUS) in 1994. From 1995 through 1996, he was a lecturer in the Department of Computer Science, Open University Degree Programme (Singapore). He joined the Nanyang Technological University in 1996, where he is now an Associate Professor.

Dr Low held the concurrent appointment of Programme Director of the Graduate Diploma Programme in Information Communication Technology from 2002 to 2005. He was the Head of the Division of the Information Communication Institute of Singapore (ICIS) from 1 July 2005 to 31 August 2008. His current research is directed towards combinatorial optimization issues that arise in communication networks with particular emphasis in the following areas: Resilience and survivability issues in wireless networks, Routing in multi-hop, ad hoc and sensor networks, and Wireless network topology adaptive control. To date he has published over 80 technical papers, some of which have appeared in the IEEE Transactions on Computers, IEEE Transactions of Parallel \& Distributed Systems, IEEE Transactions on Computer-Aided Design of Integrated Circuits and Systems, IEEE Journal of Selected Areas in Communications, IEEE Communications Letters and Information Processing Letters. Dr Low is an Associate Editor of the International Journal of Communication Systems and a Senior member of the IEEE. 